\title{
Technisch-ökonomische Zeitfenster nutzen
}

\section{Bei Wohngebäuden liegt ein Zeitfenster zu stärkeren Verbreitung energetisch anspruchsvoller Typen vor. Die nötigen Technologien sind vorhanden, erprobt und in Nischen am Markt. Was bislang fehlt, ist eine konsequente Nutzung des Fensters durch die Politik. Eine geeignete Strategie wird im Folgenden erläutert.}

$\mathrm{E}$

Von Rüdiger Haum und Jan Nill nergetisch anspruchsvolle Wohnhäuser reduzieren durch verbesserte Wärmedämmung und spezielle Lüftungsgeräte den Bedarf an Heizenergie drastisch. Innovative Alternativen zu konventionellen Wohnhäusern sind technologisch realisierbar und werden bereits gebaut, sind aber noch Nischenprodukte. Momentan existieren eine ganze Reihe von Haustypen und entsprechenden technologischen Pfaden, die durch unterschiedliche Kombinationen von energieeffizienten Technologien sowie der Nutzung von solarer Wärmegewinnung unterschiedliche Energieverbräuche vorweisen. Aus ökonomischer Perspektive relevant sind vor allem drei Pfade:

- Konventionelle Häuser nach dem Standard der Energieeinsparverordnung haben als alter Pfad einen Heizwärmebedarf von 70 bis 110 Kilowattstunden pro Quadratmeter Wohnfläche und Jahr.

- Passivhäuser als neuer Pfad, die mittels Neukombination von (passiver) Nutzung solarer Wärmegewinne, besserer Dämmung und einer Lüftungsanlage mit Wärmerïckgewinnung mit einem Heizwärmebedarf von $15 \mathrm{kWh} / \mathrm{qm}$ im Jahr und so potenziell ohne konventionelles Heizsystem auskommen.

- Dazwischen liegen Dreiliterhäuser, die als Weiterentwicklung des alten Pfades durch Integration mancher Elemente des neuen Pfades (Hybrid) einen Heizwärmebedarf von 30 bis $35 \mathrm{kWh} / \mathrm{qm}$ erreichen.

Ein techno-ökonomisches Zeitfenster für einen Wettbewerb zwischen alten und neuen Pfaden, der letzteren die Chance für Schritte aus der Nische gibt, ist derzeit gegeben. In den Bereichen Fenster, Dämmung und Lüftung gibt es eine ganze Reihe von Alternativen zu den bei herkömmlichen Gebäuden eingesetzten Technologien. Einer marktgetriebenen Verwirklichung des Zeitfensters stehen noch Schranken im Weg. Noch sind die Investitionsko- sten bei Niedrigstenergiehäusern etwa zehn bis 15 Prozent höher, je nach Haustyp und Technologieeinsatz, als bei konventionellen Häusern. Ein kostenseitiger Indikator für das Vorliegen eines technisch-ökonomischen Zeiffensters sind die annähernd vergleichbaren Gesamtkosten über den Lebenszyklus der Gebäude bei Inanspruchnahme der Fördermaßnahmen.

\section{- Kostensenkungen möglich}

Deutliche Kostensenkungen durch Innovationen und dynamische Lerneffekte insbesondere bei Fenstern und kombinierten Lüftungs- und Restheizungsgeräten sind Voraussetzung, um ihre Verbreitung weiter voran zu treiben. Die Mehrkosten könnten beispielsweise bei Fenstern nach Ansicht von Fachleuten von 50 bis 100 Prozent auf 20 bis 30 Prozent sinken.

Als Schritt in Richtung Fensternutzung hat die Kreditanstalt für Wiederaufbau (KfW) zwei Förderprogramme aufgelegt. Sie unterstuitzen Passivhäuser sowie Niedrigstenergiehäuser mit einem Jahresprimärenergiebedarf von maximal 40 oder $60 \mathrm{~kW} / \mathrm{h}$ pro Quadratmeter Wohnfläche - ersterer entspricht grob Passivhäusern, letzterer grob Dreiliterhäusern. Als Signal für eine aktive politische Nutzung des Zeitfensters ist eine Vereinbarung im Koalitionsvertrag $2003 \mathrm{zu}$ sehen, in dem eine verstärke Förderung von Passivhäusern vereinbart wurde. Die Vereinbarung führte jedoch nur zur Aufstockung der KfW-Förderung.

Eine Beispielrechnung anhand eines kosteneffizienten Passivhauses zeigte, dass die momentane Förderung die Mehrkosten fast abdeckt: Bei moderat steigenden Energiepreisen und Förderung sinken die annualisierten Mehrkosten von 642 auf 2 Euro pro Jahr und Quadratmeter (1). Angesichts der weiteren Hemmnisse bedarf es für eine effektive politische Nutzung des Zeitfensters aber weiterer Maßnahmen.

\section{Eigenheimzulage ökologisieren}

Eine Möglichkeit ist die Ökologisierung der Eigenheimzulage. Sie könnte durch die vollständige Bindung der (reduzierten) Zulage an einen Primärenergieverbrauch von höchstens $60 \mathrm{kWh}$ pro Quadratmeter bei der Errichtung von Neubauten erfolgen. Für die Erreichung des $40 \mathrm{kWh}$-Standards wäre als Signal für den Neu-Neu-Technologiewettbewerb eine deutlich höhere Fördersumme vorzusehen. Diese Chance einer gleichzeitigen Ökologisierung und Reduzierung der Eigenheimzulage wurde jedoch im Rahmen der Subventionsabbaudebatte nicht genutzt.

Alternativ empfiehlt sich eine Stärkung der KfWFörderprogramme. Die Förderung sollte stärker kommunikativ und beratend begleitet werden. Auch sollte ein Qualitätsnachweis in der Förderung verankert werden, um die Funktionserfïllung zu erhöhen und das Vertrauen in die Qualität der Häuser zu stärken. Sollten weitere politische Maßnahmen zur Nutzung des Fensters oder starke Energiepreisveränderungen ausbleiben, ist es wahrscheinlich, dass Niedrigstenergiehäuser auch langfristig Nischenprodukte bleiben.

Das Beispiel Wohngebäude zeigt, dass die zeitstrategische Analyse des Innovationsgeschehens technisch-ökonomische Zeitfenster markieren kann, auf deren Grundlage politische Zeitstrategien entwickelt werden können. In dezentralen Märkten mit niedrigen Markteintrittsbarrieren, die vielfältige technische Lösungen von Nachhaltigkeitsproblemen und ökologische Nischen erlauben, scheinen Politiken zur Fensternutzung von stärkerer Relevanz zu sein als solche zur Fenstervorbereitung; auch Subventionen können hier eine wichtige Rolle spielen (2).

\section{Anmerkungen}

(1) Haum, R./ Nill, J.: Zeitstrategien ökologischer Innovationspolitik bei Wohngebäuden. IÖW-Broschüre, Berlin 2004. (2) Nill, J.: Instrumentation of Time Strategies for an Ecological Innovation Policy: a New Role for Subsidies? IÖW-Diskussionspapier Nr. 61/04, Berlin 2004.

\section{Die Autoren}

Rüdiger Haum und Jan Nill sind wissenschaftliche Mitarbeiter im Forschungsfeld Umweltökonomie und -politik am Institut für ökologische Wirtschaftsforschung (IÖW).

Kontakt: Rüdiger Haum, IÖW, Potsdamer Str. 105, 10785 Berlin. Tel. 030-8845940, E-Mail: ruediger.haum@ioew.de 
(c) 20I0 Authors; licensee IÖW and oekom verlag. This is an article distributed under the terms of the Creative Commons Attribution Non-Commercial No Derivates License (http://creativecommons.org/licenses/by-nc-nd/3.o/), which permits unrestricted use, distribution, and reproduction in any medium, provided the original work is properly cited. 